\title{
Blastomycose disséminée entraînant la destruction de la scapula
}

\author{
Hongru Ren MD, Brett Memauri MD, Aditya Sharma MD
}

— Citation : CMAJ 2021 May 25;193:E770. doi : 10.1503/cmaj.201177-f

Voir la version anglaise de l'article ici : www.cmaj.ca/lookup/doi/10.1503/cmaj.201177

$\mathbf{U}$

n Manitobain de 38 ans atteint de diabète et d'hypertension est venu consulter au service des urgences pour une masse de plus en plus douloureuse à l'épaule droite, présente depuis 5 mois. Il a également indiqué avoir d'autres symptômes, soit de la fièvre, de la dyspnée, de la toux, des sueurs nocturnes et une perte de poids depuis 3 semaines. Il présentait un effort respiratoire légèrement accru et une tachycardie, mais sa tension artérielle et sa saturation en oxygène étaient normales. Une masse douloureuse et fluctuante de $15 \mathrm{~cm} \times 15 \mathrm{~cm}$ recouvrait la partie postérieure de sa scapula droite. Une radiographie thoracique a mis en évidence une atteinte micronodulaire diffuse et une destruction importante de la scapula droite (annexe 1, accessible en anglais au www.cmaj.ca/lookup/doi/10.1503/ cmaj.201177/tab-related-content). D'autres examens d'imagerie ont montré une masse lobulée multifocale envahissant et détruisant la scapula droite, ainsi que des lésions miliaires bilatérales diffuses (figure 1 et annexe 1). Notre diagnostic différentiel comprenait la tuberculose, une pneumonie virale, une maladie fongique disséminée et une tumeur métastatique. Nous soupçonnions également une blastomycose étant donné que le patient avait visité une région endémique au nord-ouest de l'Ontario (Kenora), où l'incidence annuelle de cette affection est de 17 cas pour 100000 personnes ${ }^{1}$. Un lavage bronchoalvéolaire et une aspiration de la masse ont révélé la présence d'une levure bourgeonnante à base large correspondant à Blastomyces dermatitidis. Nous avons prescrit une perfusion continue d'amphotéricine $B$ liposomique ainsi que le drainage échoguidé de l'abcès. Au deuxième jour du traitement, le patient a présenté une insuffisance respiratoire hypoxémique qui a nécessité un bref soutien respiratoire par pression positive biphasique. Après 1 semaine, l'amphotéricine $B$ a été changée pour du voriconazole administré par voie orale. Le traitement a duré 12 mois au total, et le patient a eu un bon rétablissement fonctionnel entre autres grâce à de la physiothérapie continue ${ }^{2}$.
Des symptômes extrapulmonaires de blastomycose surviennent chez $25 \%-40 \%$ des patients atteints d'une maladie pulmonaire, et ils touchent généralement la peau et les $\mathrm{os}^{2,3}$. Chez ces patients, les facteurs de risque d'insuffisance respiratoire nécessitant une ventilation mécanique comprennent le diabète et une maladie pulmonaire diffuse ${ }^{3,4}$. Notre cas montre l'importance de soupçonner une blastomycose chez les patients qui présentent une ostéomyélite détruisant un os, quel qu'il soit, en particulier les patients ayant visité une région où cette affection est endémique.

\section{Références}

1. Litvinjenko S, Lunny D. Blastomycosis hospitalizations in northwestern Ontario: 2006-2015. Can Commun Dis Rep 2017;43:200-5.

2. Chapman SW, Dismukes WE, Proia LA, et al. Clinical practice guidelines for the management of blastomycosis: 2008 update by the Infectious Diseases Society of America. Clin Infect Dis 2008;46:1801-12.

3. Kralt $D$, Light $B$, Cheang $M$, et al. Clinical characteristics and outcomes in patients with pulmonary blastomycosis. Mycopathologia 2009;167:115-24.

4. Lahm T, Neese S, Thornburg AT, et al. Corticosteroids for blastomycosisInduced ARDS: a report of two patients and review of the literature. Chest 2008;133:1478-80. 
Intérêts concurrents : Aditya Sharma déclare être actionnaire de ContraFect Corporation, d'Entatis Therapeutics, de Moderna, de Pfizer et de Relief Therapeutics, indépendamment du présent travail. Aucun autre intérêt concurrent n'a été déclaré.

Cet article a été révisé par des pairs.

Les auteurs ont obtenu le consentement du patient.

Affiliation : Département de médecine interne, Université du Manitoba, Winnipeg, Man.

Propriété intellectuelle du contenu : Il s'agit d'un article en libre accès distribué conformément aux modalités de la licence Creative Commons Attributions (CC BY-NC-ND 4.0), qui permet l'utilisation, la diffusion et la reproduction dans tout médium à la condition que la publication originale soit adéquatement citée, que l'utilisation se fasse à des fins non commerciales (c.-à-d., recherche ou éducation) et qu'aucune modification ni adaptation n'y soit apportée. Voir : https://creativecommons.org/licenses/by-nc-nd/4.0/deed.fr.

Correspondance : Hongru Ren, renh@myumanitoba.ca

Les images cliniques sont choisies pour leur caractère particulièrement intéressant, classique ou impressionnant. Toute soumission d'image de haute résolution claire et bien identifiée doit être accompagnée d'une légende aux fins de publication. On demande aussi une brève explication (300 mots maximum) de la portée éducative des images, et des références minimales. Le consentement écrit du patient au regard de la publication doit être obtenu avant la soumission. 